\title{
Avaliação do acesso ao SINIMA - Sistema Nacional de Informação sobre o Meio-ambiente
}

Thiago Antunes da Silva

\section{Bacharel em Arquivologia - UFRGS}

antunes.thi@gmail.com

O artigo apresenta critérios de avaliação de fontes de informação na área da Ciência da Informação. Descreve transformações na difusão do conhecimento com o surgimento da Internet, que interfere diretamente no desenvolvimento das fontes de informação, possibilitando o acesso à informação científica de todo o mundo. Ressalta o boom informacional com o advento das redes de comunicação on-line e demonstra a importância das tecnologias de comunicação e informação como um facilitador para o acesso à informação, de modo a difundir a informação ambiental na sociedade. Apresenta a avaliação do Sistema Nacional de Informação sobre o Meio-Ambiente segundo critérios de avaliação de fontes de informação de sites, com ênfase nos critérios de facilidade de uso. Conclui que o site satisfaz as necessidades do usuário, pois obedece aos critérios propostos.

Palavras-chave: Fontes de informação, avaliação de fontes de informação, facilidade de uso, Sistema Nacional de Informação sobre o Meio-Ambiente.

\section{Evaluation of the access to SINIMA - Sistema Nacional de Informação Sobre O Meio-Ambiente (National Information System for the Environment)}

The article presents criteria for evaluation of information sources in Information Science. It describes transformations in the knowledge after the Internet, which interferes directly in the information sources, allowing the access to the scientific information from 
anywhere in the world. It points out the information boom because of the online communication networks and it shows the importance of communication and information technologies as facilitators in the access to diffuse information on the environment for the society. The evaluation of the National Information System for the Environment according to criteria for evaluation of information sources is presented, with emphasis on accessibility. It concludes that the site fulfills the user needs, since it obeys the proposed criteria.

Keywords: Information sources, information sources evaluation, accessibility, National Information System for Environment.

Recebido em 13.04.2007 Aceito em 01.10.2007

\section{Introdução}

O boom informacional provocado pelo desenvolvimento das novas tecnologias, principalmente a Internet, leva os profissionais de informação a se preocuparem com a qualidade da informação. Dentre as diversas questões que se apresentam, a discussão sobre o papel das fontes no processo de qualificação da informação merece especial atenção, pois elas são agentes ativos no enquadramento da realidade retratada na mídia.

Em uma era em que a disseminação global dos resultados de pesquisas científicas é realizada cada vez mais por meios eletrônicos, o compartilhamento do conhecimento e o acesso à informação cresceram em uma proporção jamais imaginada, devendo ser estimulados cada vez mais, pois alimentam as redes de criação e inovação e ajudam a promover pesquisas de melhor qualidade. $O$ acesso aberto à literatura científica pode contribuir para a informação, criatividade, inovação e difusão do conhecimento indispensável para a formação de uma sociedade mais consciente, proporcionando o debate sobre questões globais como o futuro do meio-ambiente. A Internet tem produzido transformações no mundo dos computadores e das comunicações, como nenhuma invenção foi capaz de fazer anteriormente. Ela é uma ferramenta de disseminação da informação e um meio para a colaboração e a interação entre indivíduos e seus computadores, independentemente de suas posições geográficas.

Primeiramente, são apresentadas algumas transformações na difusão do conhecimento, com o advento da Internet. Em seguida, a atenção é voltada para a avaliação de fontes de informação na Internet e sua colaboração para a difusão de informação, quando se apresentam alguns critérios de avaliação localizados na literatura específica. Por fim, faz-se uma análise da acessibilidade do site do Sistema Nacional de Informação Ambiental, com base nesses critérios. 


\section{Transformações da difusão do conhecimento com o advento da Internet}

No contexto da sociedade, a informação é um elemento essencial, pois é por meio das trocas informacionais que os indivíduos se comunicam e tomam conhecimento de seus direitos e deveres e, a partir deste momento, tomam decisões sobre suas vidas, seja de forma individual, seja de forma coletiva. Assim, ao participarem de redes comunicacionais, os indivíduos constroem as práticas de trocas de informação. Estas podem ser conceituadas de ações de recepção, geração e transferência de informação, desenvolvendo-se em redes comunicacionais que ocorrem nas formações sociais.

Diante destas colocações, consideramos que a informação deve ser vista como um bem social e um direito coletivo como qualquer outro, sendo tão importante quanto o direito à educação, à saúde, à moradia, à justiça e tantos outros direitos do cidadão. Os conteúdos informacionais nas redes eletrônicas são analisados na perspectiva de seu impacto social e da promoção da identidade cultural. O vertiginoso desenvolvimento das tecnologias de informação e comunicação tem sido um poderoso instrumento para a rotinização, reorganização e automatização do trabalho intelectual. É da própria natureza dos elementos estruturais da sociedade da informação, sobretudo pelo avanço extraordinário da convergência tecnológica entre informática, comunicações e eletrônica, a falta de controle da produção e circulação de conhecimento.

Mais importante do que isto, contudo, é o fato incontestável de que a falta de controle da produção e circulação do conhecimento está imbricada na cultura contemporânea. Esta, por meio das tecnologias de informação e comunicação, realiza e radicaliza os sonhos de consumo do ser humano. Um dos principais indicadores do desenvolvimento do acesso à informação é a capacidade de penetração das tecnologias de informação na vida diária das pessoas e no funcionamento e transformação da sociedade como um todo, através do constante desenvolvimento das tecnologias para celular, televisão digital, mp3 e computadores.

A comunicação e a informação tendem a influenciar as atividades e os processos de decisão nas diferentes esferas da sociedade, incluindo-se a política, os governos federal, estaduais e municipais, a cultura e as artes, a ciência e a tecnologia, a educação em todas as suas instâncias, a saúde, a indústria, as finanças, o comércio e a agricultura, as associações comunitárias, as sociedades profissionais, os sindicatos, as manifestações populares, as minorias, as religiões, e a proteção do meio ambiente. Uma das contribuições da Internet é permitir que qualquer usuário, em caráter individual ou institucional, possa vir a ser produtor, intermediário e usuário de informação. E o alcance da informação é infinito, resguardadas as barreiras lingüísticas e tecnológicas do processo de difusão. 
A força motriz da formação e disseminação destas redes reside na eficiência das decisões coletivas e individuais. A Internet está sendo um canal para a veiculação de conteúdos tradicionais e também de novos formatos - tais como blogs, listas de discussões etc. - que têm peculiaridades e usos específicos, tanto de forma isolada quanto combinada. Será necessário familiarizar-se com cada um desses tipos, conhecer suas potencialidades de utilização para fins determinados e desenvolver formas adequadas de identificação e controle do ponto de vista técnico. A descrição correta desses itens informacionais é condição para seu adequado registro e entrada de dados nos sistemas de informação, premissa básica para garantir uma recuperação mais pertinente. Segundo dados do Comitê Gestor da Internet no Brasil, o acesso à Internet cresceu de 2005 para 2006, como podemos observar no gráfico abaixo (GRAF. 1):

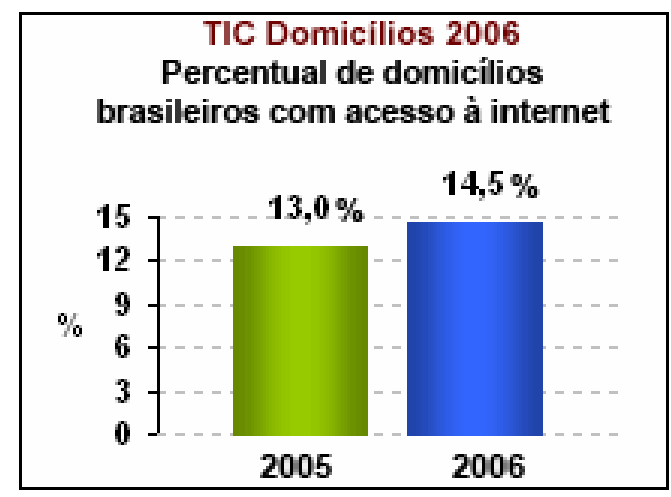

GRÁFICO 1 - Percentual de domicílios brasileiros com acesso à Internet. FONTE - CETIC (2007).

O que é importante ressaltar é que essas fontes de informação compreendem, como já foi mencionado, diferentes formatos - tais como artigos científicos, dados estatísticos, descrições de produtos etc. oriundos de diversos e variados suportes (papel, microfilme, vídeo, etc.) que necessitam de normas próprias para sua devida organização e tratamento nas tarefas de armazenamento e recuperação. A identificação desses tipos e seu controle técnico poderão facilitar as tarefas de organização dos estoques e de construção de serviços tais como centros de referência informacionais, portais e uso em conferências eletrônicas ou acesso a documentos, dentre outros.

Tanto a informação já armazenada quanto a que continua sendo produzida requerem um tratamento adequado para sua incorporação à rede, em um esforço de integração de recursos e de promoção de seu compartilhamento responsável. O que implica garantir a disponibilidade dos acervos de instituições (bibliotecas, arquivos, museus, coleções particulares etc.) como de todo e qualquer agente social produtor de informações, mediante seu tratamento técnico requerido pelo 
sistema. Requer ainda a montagem de um esquema de acessibilidade que, mesmo facilitado pela infra-estrutura da rede, requer a celebração de acordos e normas para sua distribuição e uso. (MIRANDA, 2000, p.86-87, grifos do autor).

O desenvolvimento de conteúdos na Internet brasileira deverá cobrir, ao longo dos próximos anos, todas as instâncias e atividades sociais, à medida que o governo, as organizações sociais de caráter público e privado, assim como comunidades e indivíduos, passem a operar na Internet suas demandas e ofertas de informação. É através do uso contínuo dos conteúdos em rede, a sua produção, inovação, veiculação e intermediação no dia-a-dia das instituições e dos indivíduos, que a sociedade da informação vai se conformando. Por outro lado, instituições de caráter público governamental e não governamentais, em todas as áreas, são chamadas a operar na Internet conteúdos relacionados direta e indiretamente com o fortalecimento da presença e integração do Brasil; gerando o aperfeiçoamento da democracia e a igualdade social, a cidadania, a preservação e o desenvolvimento de nossa cultura e história, o fomento ao desenvolvimento econômico, a proteção ao meio ambiente, o fomento à pesquisa científica nacional e a ampla comunicação dos seus resultados, a melhoria da saúde e da educação e, em geral, da qualidade de vida dos brasileiros.

Foi no período Pós-Segunda Guerra (1945) que os países desenvolvidos começaram a fazer grandes investimentos em tecnologias de informação e comunicação. Esse fato impulsionou o boom informacional, transformando o modo de construir e distribuir informação. Segundo Cendóm (2003), foi na década de 60, durante a Guerra Fria, que o Departamento de Defesa Norte-Americano iniciou a criação de uma rede experimental de computadores com o objetivo de aperfeiçoar as estratégias de guerra, o que resultou na criação da Advanced Research Projects Agency Network (ARPANET), em 1975, precursor da rede internacional de computadores, a Internet. Criada inicialmente para fins científicos e acadêmicos, a Internet surgiu para a população há aproximadamente vinte anos atrás, e revolucionou o acesso à informação. Em se tratando de busca de informação, as fontes de informação adquiriram um alcance incomensurável com o advento da Internet, no que tornou essencial a formulação de critérios que avaliem as qualidades das fontes. A seguir, serão apresentados alguns estudos a respeito.

\section{Critérios de avaliação de fontes de informação}

As fontes de informação são o resultado da produção do conhecimento. O desenvolvimento das tecnologias de comunicação e informação aplicadas à Internet proporcionou o crescimento do acesso às fontes de informação de tal maneira que através de um click se pode acessar pesquisas desenvolvidas no mundo inteiro. $O$ volume informacional na rede cresce diariamente, no entanto, de forma 
descontrolada e irracional, dificultando o acesso à informação. As ferramentas de pesquisa auxiliam o usuário na busca da informação desejada, mas ainda carecem de meios para avaliar a qualidade da informação pesquisada na rede, mesmo porque não é uma prática usual a estipulação de critérios de qualidade para sites. Apesar da grande quantidade, a localização de uma fonte de informação específica e eficiente na Internet pode ser uma difícil tarefa para aqueles que não levam em consideração critérios de qualidade e aspectos relacionados ao custo de busca e obtenção da informação. Segundo Tomaél et al. (2004), custo de acesso, custo de uso, custo de serviços diferenciados, energia e tempo, são exemplos de custos referentes à busca e obtenção de informações na Internet. Parizotto (1997) realizou um estudo para elaborar um guia de estilos destinado a projetistas de sites acadêmicos e de ciência e tecnologia.

Estes tipos de sites são fontes de informação constantemente utilizadas, não somente por membros da comunidade acadêmica, mas também por pesquisadores e profissionais dos mais variados segmentos. Para o referido estudo, Parizotto (1997) focou aspectos gráficos, dando ênfase ao projeto visual, tais como: layout, cores, fundos, fontes, textos e ícones. A autora afirma que o layout de uma página na Internet não exerce apenas uma função estética, mas principalmente influencia a maneira como o usuário percebe e sente a informação ali disponível. Funciona como uma importante ferramenta para auxiliar na comunicação escrita. Parizotto (1997) disserta também a respeito das vantagens e desvantagens referentes ao uso das cores em uma página da Internet, atribuindo a elas grande importância. Assim como Parizotto (1997), Tomaél et al. (2004) descrevem os dez erros no desenvolvimento de projetos de páginas na Internet, a saber: uso de frames, tecnologia inadequada devido à facilidade de aquisição, páginas muito longas, excesso de animações, URLs complexas, páginas soltas, falta de apoio para navegação, links sem padronização de cores, informações desatualizadas e páginas que demoram muito tempo para carregar.

Após estudos teóricos e pesquisas em páginas da Internet, acompanhados de testes em projeto piloto, Tomaél et al. (2004) apresentaram dez itens pormenorizados em subitens destinados à avaliação de fontes de informação na Internet. São eles: informação de identificação, consistência das informações, confiabilidade das fontes, links, facilidade de uso, layout da fonte, restrições percebidas, suporte ao usuário e outras observações recebidas. O critério de facilidade de uso desenvolvido por Tomaél (2004, p. 10-11) divide-se nos subcritérios descritos a seguir:

a-Links: que possibilitem fácil movimentação, que possibilitem avançar e retroceder; 
b-Quantidade de cliques para acessar a fonte e a informação: da página inicial até a fonte são recomendados três cliques, da fonte à informação são três ou menos cliques;

c-Disponibilidade de recursos da pesquisa na fonte: função de busca, lógica booleana, índice, arranjo, outros;

d-Recursos auxiliares à pesquisa: tesauros, listas, glossários, mapa do site, guia, ajuda na pesquisa, instruções de uso; e

e-Manuais da fonte de informação para download ou impressão.

De acordo com a NBR 9241-11, usabilidade é a "medida na qual um produto pode ser usado por usuários específicos para alcançar objetivos específicos com eficácia, eficiência e satisfação em um contexto específico de uso" (ASSOCIAÇÃO..., 2002, p. 03).

A usabilidade ou facilidade de uso na Internet constitui em elemento fundamental para o estabelecimento de redes de comunicação e informação mais eficazes, como se pode observar a seguir:

La usabilidad coloca al ser humano en el centro de la tecnología $y$, en la Infoera, se transforma en una propiedad fundamental de las interfases informáticas debido a que su orientación normalmente resulta en hacer más eficiente el acceso a la información, facilitar el proceso de construcción del conocimiento, agilizar (optimizar) la realización de las tareas, mejorar los procesos de producción y de comunicación y hacer más satisfactoria y menos frustrante la utilización de la máquin (MARTINEZ, 2005, p. 07, grifos do autor).

A usabilidade na Internet é uma área importante e em plena expansão, pois trata especificamente da relação do acesso do usuário às fontes de informação, sendo que os sites de baixa qualidade neste quesito tendem a dificultar a recuperação da informação desejada, de modo que a avaliação de sites segundo critérios cuidadosamente estudados possibilita tanto ao produtor como ao usuário da informação o aprimoramento da qualidade da informação.

\section{Avaliação do site do Sinima}

O Sistema Nacional de Informação sobre Meio Ambiente - Sinima é o instrumento da Política Nacional de Meio Ambiente, com base na Lei 6.938/1981, responsável pela gestão da informação ambiental no âmbito do Sistema Nacional de Meio Ambiente - Sisnama, de acordo com a lógica da gestão ambiental compartilhada entre as três esferas de governo.

O Sinima é coordenado pela Secretaria Executiva do Ministério do Meio-Ambiente, instituída pelo Decreto 5.776/2006, através do Departamento de Articulação Institucional - DAI, e possui três eixos estruturados: o desenvolvimento de ferramentas de acesso à informação baseadas em programas computacionais livres; a sistematização de 
estatísticas e elaboração de indicadores ambientais; a integração e interoperabilidade de sistemas de informação de acordo com uma Arquitetura Orientada a Serviços - SOA. Este processo de implantação conta com o apoio do Comitê Gestor do Sinima, instituído pela Portaria no 310 , de 13 de dezembro de 2004, no sentido da definição das diretrizes, acordos e padrões nacionais para a integração da informação ambiental. 0 site teve 14.373 acessos do período de 31/07/2006 até o dia 06/04/2007.

\subsection{Avaliação da usabilidade do sistema}

A metodologia aplicada consistiu em acessar o site durante cinco dias para avaliá-lo de acordo com os cinco subcritérios de Tomaél (2004) sobre facilidade de uso, explorando-se todos os recursos possíveis no site. Avaliou-se a ocorrência ou não do critério para cada item pesquisado. Elaborou-se um quadro (QUADRO 1) para facilitar a visualização da análise:

QUADRO 1 -Critérios para facilidade de uso de Tomaél

\begin{tabular}{|c|c|}
\hline Critérios para Facilidade de Uso & Ocorrência \\
\hline 1. Links & sim/não \\
\hline 2.Recomendação máxima de três cliques para acessar a fonte de informação & sim/não \\
\hline 3. Disponibilidade de recursos de pesquisa na fonte & sim/não \\
\hline 3.1 Função de busca & sim/não \\
\hline 3.2 Lógica booleana & sim/não \\
\hline 3.3 Índice & sim/não \\
\hline 3.4 Arranjo & sim/não \\
\hline 4. Recursos auxiliares à pesquisa & sim/não \\
\hline 4.1 Tesauros & sim/não \\
\hline 4.2 Listas & sim/não \\
\hline 4.3 Glossários & $\operatorname{sim} /$ não \\
\hline 4.4 Mapa do site & sim/não \\
\hline 4.5 Guia & sim/não \\
\hline 4.6 Ajuda na pesquisa & sim/não \\
\hline 4.7 Instruções de uso & sim/não \\
\hline 5. Manual da fonte de informação para download ou impressão & sim/não \\
\hline
\end{tabular}
FONTE - TOMAÉL (2004).

A página inicial do site contém 23 links, sendo que nove tratam diretamente do Sinima e os restantes são relacionados ao assunto meioambiente. Como podemos observar abaixo (FIG. 1), no centro da página localizam-se três links específicos para a recuperação da informação: 


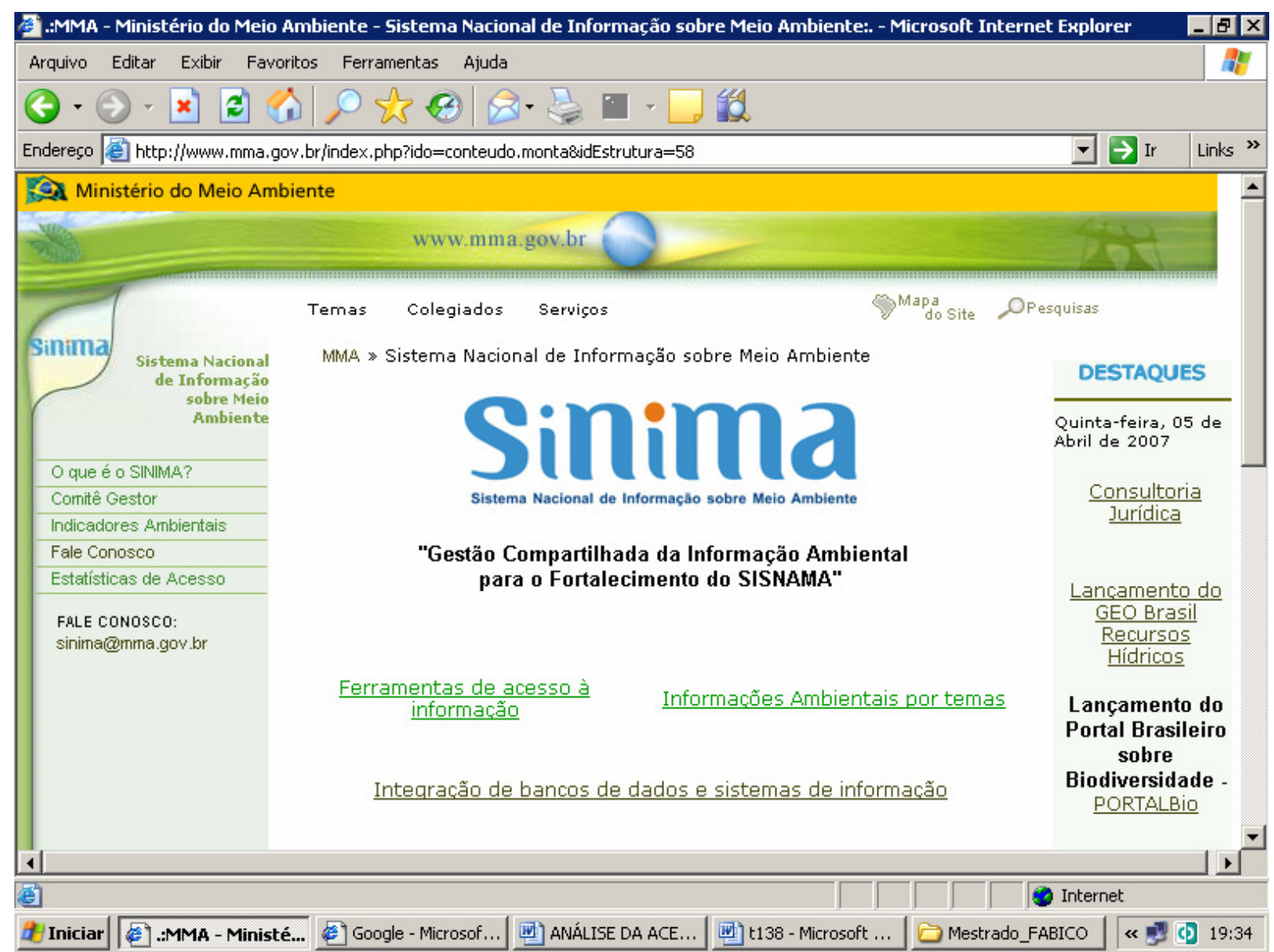

FIGURA 1 - Página inicial do site Sinima.

FONTE - BRASIL (2007).

Em Ferramentas de acesso à informação, encontram-se três links de pesquisa: o geoprocessamento, que é um instrumento para obtenção, processamento e difusão de dados espaciais, contendo mapas e imagens de satélites; a localização por meio de coordenadas geográficas, sendo uma base de dados que, através da checagem de coordenadas, recupera informação sobre municípios, regiões hidrográficas, bacias hidrográficas, biomas, unidades de conservação federais e outros dados geográficos; o Cliente $\mathrm{R}$, que é um programa estatístico para a realização de cálculos para gerar indicadores ambientais.

Em Informações Ambientais por Temas encontram-se seis links tratando de assuntos gerais sobre meio-ambiente, que são: informações municipais, áreas prioritárias para a conservação, terras indígenas, unidades de conservação federais, licenças ambientais e poços de água. A seguir, analisa-se cada item pormenorizadamente:

1. Informações municipais - este link contém mapas de imagem, relevo e municípios de todo o Brasil. A pesquisa do município pode ser feita de duas maneiras. A primeira, através de uma lista dos estados do Brasil, em que cada estado é um link. Gera-se uma relação em ordem alfabética de todos os municípios do estado, 
sendo que cada município é um link. Na segunda, digitase o nome do município a ser pesquisado, logo abaixo da lista de estados, e clica-se no botão "procurar" para gerar o resultado da busca. As informações disponíveis em forma de link são: análise municipal, terras indígenas, unidades de conservação federais, áreas de prioridade de conservação de biodiversidade, meio físico/biótico e Agenda 21.

2. Áreas prioritárias para a conservação - mapa que demonstra a prioridade de conservação de acordo com uma escala que vai de "extremamente alta" a "insuficientemente conhecida". Cada grau da escala de prioridade é um link, que contém uma lista de todas as áreas de conservação no mapa.

3. Terras indígenas - mapas que mostram as áreas indígenas segundo a seguinte classificação: confirmada, declarada, delimitada, homologada e regularizada.

4. Unidades de conservação federais - listagem de mapas divididos em: proteção integral federal, uso sustentável federal, proteção integral estadual e uso sustentável estadual.

5. Licenças ambientais - mesmo sistema de busca de Informações municipais.

6. Poços de água - mesmo sistema de busca de Informações municipais e Licenças ambientais.

Em Integração de Bancos de Dados e Sistemas de Informação localizam-se links para outros portais e sistemas de informação relacionados ao meio ambiente. São eles:

Portal Nacional do Licenciamento Ambiental - PNLA

Portal Brasileiro sobre Biodiversidade - PORTALBio

Bases Compartilhadas de Dados sobre a Amazônia - BCDAM

Rede Virtual da Caatinga - RVC

Sistema de informações do Gerenciamento Costeiro e Marinho Sigercom

Sistema de informações do Rio São Francisco - SisFran

Sistema Brasileiro de Informações sobre Educação Ambiental SIBEA

Sistema de Informações Ambientais no Mercosul - SIAM

Sistema de Georreferenciamento de Projetos - Sigepro

Este link em particular é muito útil do ponto de vista de pesquisadores e usuários em geral, pois amplia o universo de buscas de fontes de informação relacionadas ao tema, possibilitando o 
aproveitamento de informações de diferentes bases de dados e sistemas de informações.

\subsection{Resultados da avaliação}

Após cinco dias de análise da usabilidade do Sinima - Sistema Nacional de Informação sobre o Meio-Ambiente, constatou-se que o site obedece plenamente ao critério Facilidade de uso, sendo todos os links acessíveis com menos de três clicks para se chegar à fonte de informação. O site contém recursos de pesquisa diversos, como ferramentas de acesso à informação geográfica, links temáticos e bases de dados integradas, estando todos localizados na página inicial do site. Quanto à ocorrência de recursos auxiliares de pesquisa, o site disponibiliza um link denominado Pesquisas, que é uma página que contém três tipos de pesquisa: (1) pesquisa na página do site do Ministério do Meio-Ambiente (MMA), através de uma barra de pesquisa Google, com opções de busca simples e avançada; (2) link para pesquisa do Ibama, no Centro Nacional de Informação Ambiental; e (3) busca no Portal de Serviços e Informações do Governo Federal. Não há ocorrência de tesauros e de glossários. O mapa do site ocorre na forma de link em todas as páginas que se abre, configurando-se como um menu principal dividido em Temas, Serviços, Colegiados e Entidades Vinculadas, estes subdivididos em assuntos mais específicos, constituindo um índice de fácil visualização e utilização. Quanto à existência de manual das fontes de informação para download ou impressão, não se registrou a ocorrência. Abaixo observamos os resultados da aplicação dos critérios de avaliação:

QUADRO 2- Critérios para facilidade de uso

\begin{tabular}{l|l}
\hline \multicolumn{1}{c|}{ Critérios para Facilidade de Uso } & Ocorrência \\
\hline 1. Links & sim \\
\hline 2. Recomendação máxima de três cliques para acessar a fonte de informação & sim \\
\hline 3. Disponibilidade de recursos de pesquisa na fonte & sim \\
\hline 3.1 Função de busca & $\operatorname{sim}$ \\
\hline 3.2 Lógica booleana & $\operatorname{sim}$ \\
\hline 3.3 Índice & sim \\
\hline 3.4 Arranjo & sim \\
\hline 4. Recursos auxiliares à pesquisa & não \\
\hline 4.1 Tesauros & sim \\
\hline 4.2 Listas & não \\
\hline 4.3 Glossários & sim \\
\hline 4.4 Mapa do site & sim \\
\hline 4.5 Guia & sim \\
\hline 4.6 Ajuda na pesquisa & não \\
\hline 4.7 Instruções de uso & não \\
\hline 5. Manual da fonte de informação para download ou impressão & \\
\hline FONTE - & \\
\hline
\end{tabular}

FONTE - Dados da pesquisa. 


\section{Considerações finais}

O Sinima obedece à maior parte dos critérios sobre facilidade de uso com aplicação neste trabalho, constituindo uma rede de comunicação e informação eficaz para pesquisadores e demais usuários. Mesmo tendo uma quantidade considerável de links, a navegação pelo site é facilitada por excelentes ferramentas de pesquisa, o que torna o acesso à informação rápido, mesmo para usuários leigos no tema. O Sinima está integrado a uma rede de informação maior dentro do Ministério do MeioAmbiente, que inclui o CNIA, Centro Nacional de Informação Ambiental do Ibama e entidades vinculadas, expandindo as possibilidades de pesquisa de informação ambiental na Internet. O Sinima é um bom exemplo da boa qualidade de fontes de informação produzidas pelo poder público na Internet, sendo assim seu modelo deve ser seguido no constante desenvolvimento do acesso à informação como uma prerrogativa da sociedade na direção da igualdade de direitos e do desenvolvimento da conscientização das problemáticas ambientais.

\section{Referências}

MIRANDA, A. Sociedade da informação: globalização, identidade cultural e conteúdos. Ci. Inf., v.29, n.2, maio/ago. 2000

CENDÓN, B. V. A Internet. In: CAMPELO, B. S.; CEDON, B. V.; KREMER, J. M.(Org.). Fontes de informação para pesquisadores e profissionais. Belo Horizonte: Ed. da UFMG, 2003. p. 275-300.

TOMAÉL, M. I. et al. Critérios de qualidade para avaliar fontes de informação na Internet. In: TOMAÉL, M. I.; VALENTIM, M. L. P. (Org.). Avaliação de fontes de informação na Internet. Londrina: Eduel, 2004. p. 19-40.

PARIZOTTO, R. Elaboração de um guia de estilos para serviços de informação em ciência e tecnologia via Web. 1997. 111 f. Dissertação (Mestrado em Engenharia de Produção) - Universidade Federal de Santa Catarina, Santa Catarina, 1997.

BRASIL. Decreto No 5.776, de 12 de maio de 2006. Aprova a Estrutura Regimental e o Quadro Demonstrativo dos Cargos em Comissão e das Funções Gratificadas do Ministério do Meio Ambiente, e dá outras providências. Diário Oficial da União, 15 maio 2006.

. Lei no 6.938, de 31 de agosto de 1981. Dispõe sobre a Política Nacional do Meio Ambiente, seus fins e mecanismos de formulação e aplicação, e dá outras providências. Diário Oficial da União, 02 set. 1981.

. MINISTÉRIO DO MEIO AMBIENTE. Sistema Nacional de Informação Ambiental - SINIMA. Disponível em: $<$ http://www.mma.gov.br/index.php?ido=conteudo. monta\&idEstrutura $=5$ 8\&idConteudo=4871\# > . Acesso em: 04 ago. 2007. 
COMITÊ GESTOR DA INTERNET NO BRASIL. Centro de Estudos sobre as Tecnologias da Informação e da Comunicação - CETIC. Disponível em: <www.cetic.br>. Acesso em: 08 abr. 2007.

ASSOCIAÇÃO BRASILEIRA DE NORMAS TÉCNICAS - ABNT. NBR 9241-11: requisitos ergonômicos para trabalho de escritórios com computadores. Parte 11 - orientações sobre usabilidade. Rio de Janeiro, ago. 2002. 21 p.

MARTINEZ, M. L. La importancia de la usabilidad en la era de la información: la humanización de las tecnologías. In: SEMINÁRIO INTERNACIONAL LATINO-AMERICANO DE PESQUISA DA COMUNICAÇÃO, III, São Paulo, Anais... São Paulo: ALAIC, 2005. Cd. Rom.

SILVA, T. E.; TOMAÉL, M. I. (Org.). Fontes de informação na Internet: a literatura em evidência. In: TOMAÉL, M. I.; VALENTIM, M. L. P. (Org.). Avaliação de fontes de informação na Internet. Londrina: Eduel, 2004. p. 01-17. 